\title{
PAPR Reduction of OFDM Signal for Visible Light Communication by Using Hartley Transform and Companding Techniques
}

\author{
Zhongpeng WANG ${ }^{1,}$, , Fangni Chen ${ }^{1}$, Jianfeng WENG ${ }^{1}$ \\ 1School of Information and Electronic Engneering, Zhejiang University of Science and Technology, \\ Hangzhou, 210023, China \\ ${ }^{2}$ State Key Laboratory of Millimeter Waves, Southeast University, Nanjing, 210096, China \\ aemail:wzp1966@sohu.com
}

Keywords: Visible Light Communication, DC-OFDM, precoding, PAPR, companding

\begin{abstract}
OFDM Visible light communication (VLC) systems can achieve both illumination and data transmission simultaneously via light emitting diodes (LEDs). Orthogonal frequency-division multiplexing (OFDM) has been considered as a promising multi-carrier modulation technique for VLC due to its ability to boost data rates and efficiently combat inter-symbol-interference (ISI). However, the peak-to-average power ratio (PAPR) is the main problem of the OFDM-based VLC system. In this paper, a combined discrete Hartley transform (DHT) precoding and companding transform scheme is proposed to reduce the PAPR of OFDM-based VLC systems. The performance of the proposed PAPR reduction scheme is studied via simulation. Simulation results show that the proposed scheme may obtain significant PAPR reduction compared to the previously proposed DHT spread (DHTS) and conventional companding transform (CT) in terms of PAPR reduction capability. The bit error rate (BER) performance of the OFDM-based VLC system with combined DHT precoding and companding is also evaluated over an indoor dispersive VLC channel model. The simulation shows that the BER of the proposed scheme outperforms that of the companding scheme used alone.
\end{abstract}

\section{Introduction}

In recent years, visible light communication has been attracted a growing interest and can be a promising complement candidate to conventional RF communication. VLC can provide both illumination and data transmission simultaneously via visible light [1]. The main advantage of VLC is its cost-effective, electromagnetic interference free and license-free and secure communication link. In some RF-restricted environments such as airplanes and hospitals, VLC can be a potential candidate solution. Because that Orthogonal frequency division multiplexing (OFDM) can provide greater immunity to multi-path fading and reduce the complexity of equalizers, OFDM has been researched in VLC systems. In VLC systems, intensity modulation with direct detection (IM/DD) is employed. The IM/DD based VLC requires real-valued non-negative time domain signals. To ensure that the OFDM time domain signal is real-valued, the input signal vector of the IFFT of transmitter is constrained to have Hermitian symmetry. However, the real-valued signal is still bipolar. In order to provide the non-negative signal, further processing is required. Now there are three approaches to deal with this issue, they are DC-bias optical OFDM (DCO-OFDM), asymmetric clipped optical OFDM (ACO-OFDM), and pulse-amplitude-modulated discrete multitone (PAM-DMT), respectively [2]. DCO-OFDM adds a DC offset to the real-valued signals. For ACO-OFDM, only the odd-numbered sub-carriers carry data without adding DC-bias. For PAM-DMT, only imaginary part of each subcarrier is modulated by data without adding DC-bias.

Because that illumination is one function of OFDM-based VLC, so maintaining a certain average power is required for VLC systems. Thus, DCO-OFDM based VLC is potential candidate solution for VLC applications. Without loss of generality, we will focus on DCO-OFDM in this work. We will investigate the performances of DCO-OFDM in terms of PAPR and BER. However, the major drawback of DCO-OFDM based VLC is its large peak-to-average power ratio (PAPR), which causes BER performance deterioration and affect the brightness control [3]. 
To reduce the PAPR, various techniques have been proposed for OFDM-based VLC systems, such as clipping [4], nonlinear companding transforms [5, 6], precoding transforms [7] and other techniques etc [8]. These schemes can mainly be categorized into signal scrambling techniques, such as PTS, and signal distortion techniques such as clipping, companding techniques. Among those PAPR reduction methods, the simplest scheme is to use the clipping process. However, using clipping processing causes both in-band distortion and out-of-band distortion and further causes an increasing of error bit rate of system. As an alternative approach, a companding shows better performance than clipping technique because the inverse companding transform (expanding) is applied in receiver end to reduce the distortion of signal. Discrete Hartley transform (DHT) may reduce PAPR of OFDM signal while the error probability of system is not increased. In [9, 10], a combined Hardamard and companding scheme has been proposed to reduce the PAPR of OFDM signal. Furthermore, this method has been employed in IM/DD OFDM system to reduce the PAPR of optical OFDM systems [11]. Based on the idea of [9, 10] and [11], in this paper we propose an efficient reducing PAPR technique based on joint companding and DHT precoding scheme for reduction PAPR of OFDM-based VLC systems.

\section{PAPR in OFDM-based VLC Systems}

In a VLC system, LEDS are the main source of non-linearity. The maximum amplitude of signal is limited by the dynamic range of the LEDS for an OFDM-based VLC system. Thus, the signal outside of this range will be clipped. With digital predistortion, the non-linear characteristics of LEDs can be linearized within a limited interval $\left[V_{l}, V_{u}\right]$, where $V_{l}$ denotes the lower clipping level and $V_{u}$ denotes the upper clipping level. The dynamic range can be denoted by $D \triangleq V_{u}-V_{l}$.

In an OFDM-based VLC system, binary bits are firstly mapped to quadrature amplitude modulation (QAM) symbols. Then the QAM symbol vector $X=\left[\begin{array}{llll}X_{0} & X_{1} & \ldots & X_{N-1}\end{array}\right]$ are constrained by Hermitian symmetry, which can be described as

$$
X[k]=X^{*}[N-k], \quad k=1,2, \ldots, N / 2-1,
$$

where *denotes complex conjugate. $\mathrm{N}$ is the number of subcarriers. $X[0]$ and $X[N / 2]$ are set to zero. After that, signal $\left[\begin{array}{llll}x[0] & x[1] & \ldots & x\{N-1]\end{array}\right]$ can be obtained by applying the IFFT operation to a frequency domain signal $X=\left[\begin{array}{llll}X_{0} & X_{1} & \ldots & X_{N-1}\end{array}\right]$ as

$$
x[n]=\frac{1}{\sqrt{N}} \sum_{k=0}^{N-1} X[k] e^{j 2 \pi k n / 2 \pi}, \quad 0 \leq k<N
$$

Where $\mathrm{n}$ is the discrete-time index and $j=\sqrt{-1}$. Let $\kappa_{d}$ denote the set of data-carrying subcarriers with cardinality $\left|\kappa_{d}\right|$. The set of data-carrying subcerriers of system is $\kappa_{d}=\left\{\begin{array}{llllllll}1 & 2 & \ldots & N / 2-1 & N / 2+1 & \ldots & N-2 & N-1\end{array}\right\}$ and $\left|\kappa_{d}\right|=N-2$. So the time domain signal $x[n]$ can be obtained as

$$
x[n]=\frac{2}{\sqrt{N}} \sum_{k=1}^{N / 2-1}\left(\Re\left(X_{k}\right) \cos (2 \pi k n / N)-\Im\left(X_{k}\right) \sin (2 \pi k n / N)\right)
$$

Where is real-valued. In general, the PAPR of OFDM signals $x(n)$ is defined as the ratio period between the maximum instantaneous power and its average power during an OFDM symbol. It can be written as

$$
\operatorname{PAPR}[x(n)]=10 \lg \frac{\max _{0 \leq n \leq N}\left[|x(n)|^{2}\right]}{E\left[|x(n)|^{2}\right]},
$$

where $E[\cdot]$ stands for the statistical expectation. The PAPR of OFDM signal usually be evaluated via complementary cumulative distribution function (CCDF) of the PAPR, which is defined by 


$$
P C=\mathrm{P}\{P A P R>P A P R 0\},
$$

where Pc indicates the probability that PAPR exceeds a particular value PAPR0.

\section{System Principle of the Proposed Scheme}

OFDM in VLC systems inherits the disadvantage of high peak-to-average power ratio (PAPR) from OFDM in radio frequency (RF) systems. Companding is an effective technique to reduce the peak-to-average power ratio of OFDM signal. Its idea is that the small signal is amplified while the large signal is compressed in order to keep signal power unchanged. In this work, the idea of companding is can be fond in [10]. In order to further reduce the PAPR of OFDM signal, a combined DHT precoding and companding scheme is proposed. In the first step of the proposed scheme, the coming data vector is firstly transformed by a DHT precoding into a new transformed data vector. After that the IFFT operation is applying to the transformed data vector. In the second step, the companding technique is employed to the output data vector of IFFT in order to further reduce the PAPR of OFDM signals. The all system block is shown at Fig. 1.

The incoming bit stream is firstly modulated using QAM signal. Then the signal vector $S=\left[\begin{array}{llll}S_{0} & S_{1} & \ldots & S_{M-1}\end{array}\right]$ is transformed by DHT precoding matrix P into new transformed signal vector, which is can be written as

Where $\mathrm{P}$ is defined as

$$
Y=P S,
$$

$$
P=\left[\begin{array}{cccc}
p_{00} & p_{01} & \cdots & p_{0(M-1)} \\
p_{10} & p_{11} & \cdots & p_{1(M-1)} \\
\vdots & \vdots & \ddots & \vdots \\
p_{(M-1) 0} & p_{(M-1) 1} & \cdots & p_{(M-1)(M-1)}
\end{array}\right],
$$

Where where $p_{i j}$ means i-th row and l-th column of DHT precoding matrix. The $p_{i j}$ is defined as

$$
p_{i j}=\operatorname{cas}\left(\frac{2 \pi i j}{M}\right)=\cos \left(\frac{2 \pi i j}{M}\right)+\sin \left(\frac{2 \pi i j}{M}\right) \text {, }
$$

where $i=0,1, \ldots, M-1$ and $j=0,1, \ldots, M-1$.Then the frequency domain signals $X=\left[\begin{array}{llll}X_{0} & X_{1} & \ldots & X_{N-1}\end{array}\right]$ can be formed and expressed as

$$
X=\left[\begin{array}{llllllll}
0, & Y_{0} & \ldots & Y_{M-1} & 0 & Y_{M-1}^{*} & \ldots & Y_{0}^{*}
\end{array}\right]
$$

After an N-point IFFT operation is applied to $X$, we can obtain the real-valued time domain OFDM signal $x[n]$ as

$$
x[n]=\frac{2}{\sqrt{N}} \sum_{k=1}^{N / 2-1}\left(\Re\left(X_{k}\right) \cos (2 \pi k n / N)-\Im\left(X_{k}\right) \sin (2 \pi k n / N)\right)
$$

Then, the output signal of IFFT is compressed and the resulting signal can be written as

$$
\hat{x}(n)=C\{x(n)\}=\frac{v x(n)}{\ln (1+u)|x(n)|} \ln \left(1+\frac{u}{v}|x(n)|\right),
$$

where $v$ is the average amplitude of signal and $u$ is the companding parameter. Considering a companded OFDM system with constant transmission power, companding transform should satisfy the following two conditions:

$$
\begin{aligned}
& \text { (1) } E\left(|\hat{x}(n)|^{2}\right) \approx E\left(|x(n)|^{2}\right) . \\
& \text { (2) }|\hat{x}(n)| \geq|x(n)| \text { when }|x(n) \leq v| \text {; } \\
& |\hat{x}(n)| \leq|x(n)| \text { when }|x(n) \geq v| .
\end{aligned}
$$

However, the signal $\hat{x}[n]$ is bipolar real-valued. In order to generate a uniploar signal, a DC-bias is added to $\hat{x}[n]$. Then a resulting signal can be written as

$$
z[n]=\hat{x}[n]+B
$$

where B denotes the value of DC-bias. After D/A conversion, the signal which is regarded as input of LED is transformed into optical signal. The resulting optical signal is propagated over 
wireless optical channel.

At receiver end, the received optical signal is transformed into electronic signal by a PD component. After A/D conversion, the obtained signal $r[n]$ is expanded by the inverse companding transform before it is sent to the FFT process unit. The expanding equation is

$$
\hat{r}(n)=C^{-1}\{r(n)\}=\frac{v r(n)}{u|r(n)|}\left\{\exp \left[\frac{|r(n)| \ln (1+u)}{v}\right]-1\right\}
$$

Then FFT operation is applied to the $\operatorname{signal}^{\hat{r}(n)}$, we can obtain frequency domain signal vector $R$. After removed the Hermitian symmetry part of $R$, the resulting signal $\hat{Y}$ is transformed by an inverse DHT precoding matrix. Then the new signal can be expressed as

$$
\hat{S}=P^{H} \hat{Y}
$$

Final the signal $\hat{S}$ is demapped to bit stream.

\section{Simulation Results}

\section{PAPR performance}

In this section, computer simulations are used to evaluate the peak-to-average ratio reduction capability using CCDF parameter. Fig. 2 shows the CCDF performance comparison of proposed scheme for QPSK modulation. In simulation, the value of $u$ is fixed at 1,2 , and 3, respectively. We can see that the PAPR performance of the proposed scheme based on combined DHT precoding and companding is better than that of the DHT precoding and companding used alone. At CCDF=10-3, the proposed scheme $(\mathrm{u}=1)$ can reduce PAPR of OFDM signal almost $0.5,3.5,4.5 \mathrm{~dB}$ compared to that of companding scheme $(\mathrm{u}=1)$, DHT precoding and original OFDM, respectively. For different value of $u$ of companding, the PAPR performance of the combined DHT precoding and companding is better than that of companding technique and DHT precoding used alone.

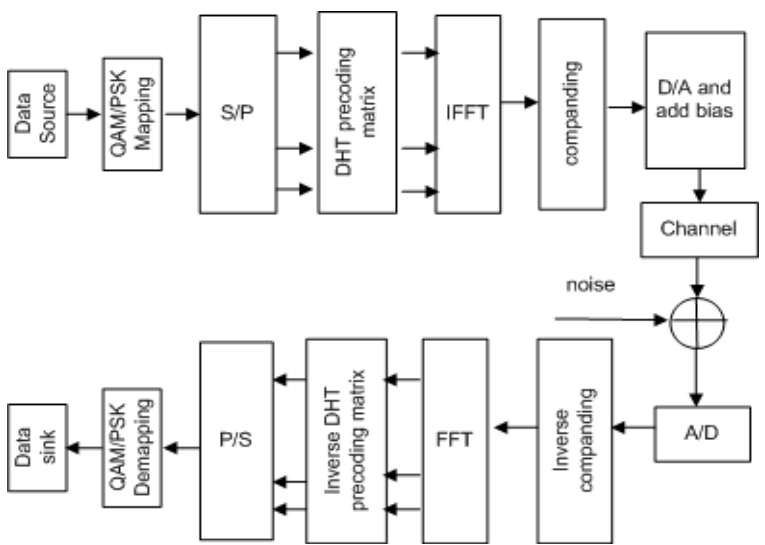

Fig.1. Block diagram of a visible light OFDM system with DHT precoding and companding

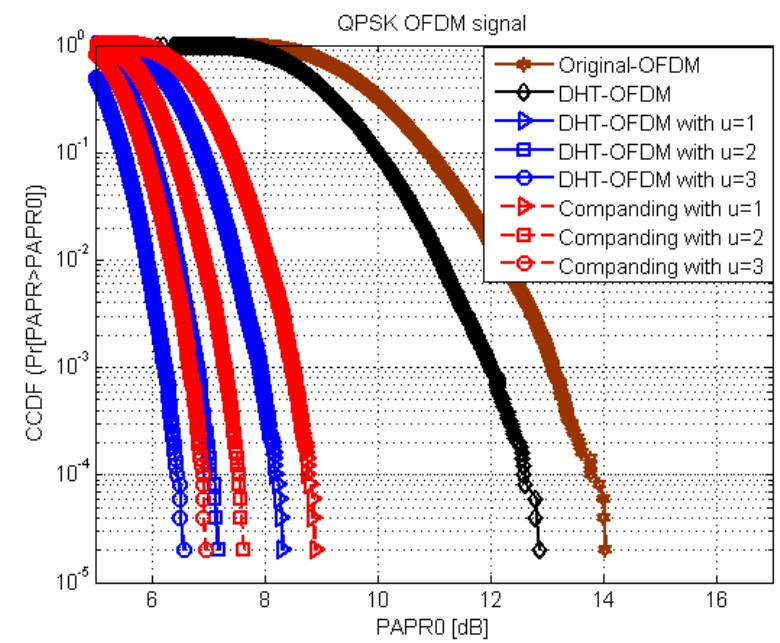

Fig. 2. Comparison of CCDF of PAPR

\section{BER performance}

Because that the ceiling-bounce model is widely used in diffused optical wireless channel, so we evaluate the BER of the proposed for the ceiling-bounce model. The continuous impulse response of an optical wireless link $h(t)$ can be represented as [9]

(17)

$$
h_{c}(t)=H(0) \frac{6 a^{6}}{(t+a)^{7}} u(t)
$$

where $a=2 H / c, H$ is the ceiling height above the transmitter, $c$ is the velocity of light. Assume $u(t)$

Table 1 Simulation Parameters

\begin{tabular}{|c|c|}
\hline Rb & 500 Mbits/s \\
\hline Modulation & QPSK \\
\hline FFT size & 256 \\
\hline Number of pilot data & 8 \\
\hline Length of CP & 32 \\
\hline$H$ & $3.5 \mathrm{~m}$ \\
\hline
\end{tabular}


be the unit step function and $H(0)$ be the channel DC gain. In our simulation, the main parameters are shown in Table 1.

Fig. 3 shows the comparison of BRR performance of several schemes over diffuse optical wireless channel. The value of $u$ of companding scheme is fixed at 1,2 and 3 . We can see that the BER performance of companding scheme is degraded compared to that of the original OFDM, DHT precoded OFDM due to signal distortion causing by companding processing. With value of $u$ increasing, the BER performance of the companding scheme is degraded. From Fig. 3 we can also see that the BER performance of companding shceme with $\mathrm{u}=1$ is better than that of companding scheme with $\mathrm{u}=2$ or 3 when SNR is lower than $24 \mathrm{~dB}$. However, when SNR is higher than $24 \mathrm{~dB}$, the BER performance of companding scheme with $u=2$ is the best.

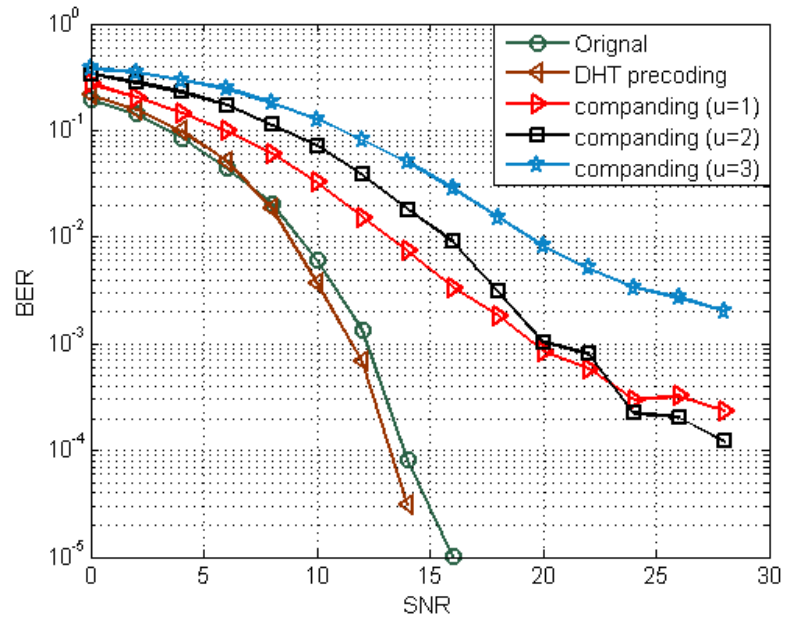

Fig. 3. Comparison of BER performance of original OFDM, DHT precoding scheme and companding scheme.

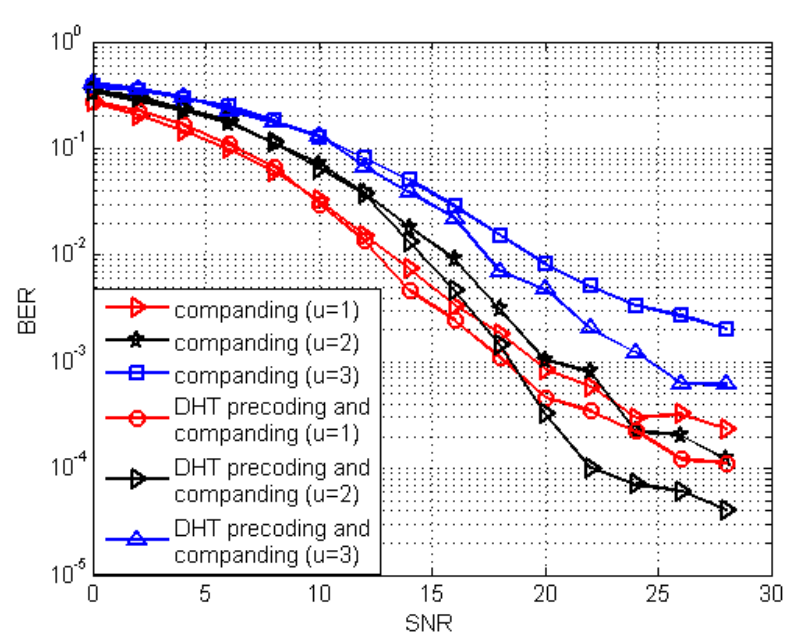

Fig. 4. Comparison of BER performance of companding scheme and combined DHT precoding and companding scheme

Fig. 4 shows the comparison of BER performance for companding scheme and combined DHT precoding and companding scheme over diffused optical wireless channel. inthe proposed scheme the value of $\mathrm{u}$ is also fixed at 1,2 and 3. We can see that the BER performance of the proposed scheme using combined DHT precoding and companding is better than that of companding scheme used alone at different values of u. From Fig. 4 we can obtain the similar results with Fig. 3, which the BER performance of the combined DHT precoding and companding scheme with $u=1$ is the best when SNR is lower than $18 \mathrm{~dB}$. The BER performance of the proposed scheme with $\mathrm{u}=2$ is better than that of the proposed scheme with $\mathrm{u}=1$.

\section{Conclusion}

In this paper, a PAPR reduction scheme based on joint DHT precoding and companding technique is proposed for OFDM-based VLC system. The PAPR reduction performance and BER performance are evaluated by computer simulation. Simulation results state that the PAPR reduction performance is improved compared with companding transform used only. On the other hand, the BER of system using proposed PAPR reduction scheme can be improved compared to that of companding used alone. 


\section{Acknowledgement}

In this paper, the research was sponsored by the Nature Science Foundation of Zhejiang Province (Project No. LY13F050005), and Open Fund of the State Key Laboratory of Millimeter Waves (Southeast University, Ministry of Education, China) (Project No. K201214).

\section{References}

[1] J. Grubor, S. Randel, K.-D. Langer, and J. Walewski. Broadband information broadcasting using led-based interior lighting [J] Journal of Lightwave Technology, 2010:26 (24): 3883-3892.

[2] Qi Wang, Zhaocheng Wang, and Linglong Dai. Iterative receiver for Hybrid asymmetrically clipped optical OFDM [J]. Journal of Lightwave Technology, 214: 32 (22): 3869-3875.

[3] H. Elgala, R. Mesleh, and H. Haas. Non-linearity effects and predistortion in optical OFDM wireless transmission using LEDs [J]. International Journal of Ultra Wideband Communications and Systems, 2009: 1 (2):143-150.

[4] ZhenHua Yu, Robert J Baxley and Guo Tong Zhou. EVM and achievable data rate analysis of clipped OFDM signals in visible light communication [J], EURASIP Journal on wireless communications and Network, 2012: 321.

[5] Kasun Bandara, Pararajasingam, Y. H. Chung. On Companding Transform Techniques for OFDM Visible Light Communication over Indoor Dispersive Channels [J]. Advances in computer and its applications, Lecture Notes in Electrical Engineering 2014 (279) 249-254.

[6] K. Bandara, P. Niroopan, Y. Chung. PAPR reduced OFDM visible light communication using exponential nonlinear companding [C]. In Proceedings of IEEE COMCAS, 2013:1-5.

[7] B. Ranjha, M. Kavehrad, Precoding techniques for PAPR reduction in asymmetrically clipped OFDM based optical wireless system [J]. SPIE OPTO. International Society for Optics and Photonics, 2013: 86450R-86450R-9.

[8] H. Zhang, Y. Yuan, W. Xu. PAPR reduction for DCO-OFDM visible light communications via semidefinite relaxation [J]. IEEE Photon, Technol. Lett. 2014:26 (17): 1718-1721.

[9] Z. Wang, S. Zhang, B. Qiu. PAPR reduction of OFDM signal by using Hadamard transform in companding techniques [C]. IEEE International Conference Communications, 2010 320-323.

[10] PAPR reduction based on precoding techniques with companding in OFDM systems, [J] International Journal of scientific \& engineering research, 2013: 4 (5): 1064-1069.

[11] Jiangnan Xiao, Jianjun Yu, Xinying Li, Qi Tang, Hongxian Chen, Fan Li, Zizheng Cao, and Lin Chen. Hadamard Transform Combined With Companding Transform Technique for PAPR Reduction in an Optical Direct-Detection OFDM System [J]. Journal of optical communications and networking, 2012: 4(10): 709-714. 